\section{Recovering Articulated Pose: A Comparison of Two Pre and Postimposed Constraint Methods}

\author{
Teofilo E. de Campos, Ben J. Tordoff, and \\ David W. Murray, Member, IEEE
}

\begin{abstract}
We contrast the performance of two methods of imposing constraints during the tracking of articulated objects, the first method preimposing the kinematic constraints during tracking and, thus, using the minimum degrees of freedom, and the second imposing constraints after tracking and, hence, using the maximum. Despite their very different formulations, the methods recover the same pose change. Further comparisons are drawn in terms of computational speed and algorithmic simplicity and robustness, and it is the last area which is the most telling. The results suggest that using built-in constraints is well-suited to tracking individual articulated objects, whereas applying constraints afterward is most suited to problems involving contact and breakage between articulated (or rigid) objects, where the ability to test tracking performance quickly with constraints turned on or off is desirable.
\end{abstract}

Index Terms-Visual tracking, articulated objects, motion constraints.

\section{INTRODUCTION}

MODEL-BASED tracking of multiple and articulated objects remains a challenge more than twenty years after Hogg demonstrated visual tracking of a walking person modeled using 3D cylinders [1]. The taxing issues remain those of how to represent the objects and their composited pose, how to associate image data with the correct part of the object, by what computational means to adjust the high-dimensional state vector to improve the fit to current observations, and how to overcome fundamental ambiguities in the observations. The variety of solutions evoked is most apparent in work to understand body motion. For representation, most use standard or generalized cylinders [1], [2], [3], but simpler planar [4] and more sophisticated quadrics-based [5], [6], [7] and deformable [8] models have been explored. The most widely used feature in matching between image and model is the edge, e.g., [5], [9], [10], [11], but increasing use is made of internal features, such as corners [12] and image motion [2], [3], [13], [14]. The key distinction in fitting pose to the image data is between works that adopt statistical techniques based on simple unimodal pdf's and solve deterministically, e.g., [2], [10], and those that represent arbitrary multimodal pdf's using mixture models or particle filters [3], [9], [15]. Overcoming ambiguities, particularly troublesome from single views, is explored in [16], [17].

The issue of concern here is whether there are compelling reasons to represent articulated pose either 1) as set of constraints imposed after each subpart is tracked independently, so introducing redundant degrees of freedom in tracking to be quenched later, or 2) as a set of built-in, preimposed constraints between each subpart of a model, thereby minimizing the degrees of freedom from the outset.

Exemplars of both classes of method have been reported in a number of application areas. In the "postimposed" class is the

- T.E. de Campos and D.W. Murray are with the Department of Engineering Science, University of Oxford, Parks Road, Oxford OX1 3PJ, UK.

E-mail: $\{t e o, d w m\} @$ robots.ox.ac.uk.

- B. Tordoff is with the Department of Engineering, Cambridge University, Trumpington St., Cambridge CB2 1PZ, UK. E-mail: bjt21@eng.cam.ac.uk.

Manuscript received 21 May 2004; revised 17 Feb. 2005; accepted 20 Apr. 2005; published online 11 Nov. 2005.

Recommended for acceptance by P. Fua.

For information on obtaining reprints of this article, please send e-mail to: tpami@computer.org, and reference IEEECS Log Number TPAMI-0254-0504. work of Drummond and Cipolla [10], [18]. They use exponential representations of motion and Lie algebra, tracking subparts independently and then applying constraints using Lagrange multipliers. Wu et al. [19] track hands by imposing constraints on independent subparts via a Markov network. Hel-Or and Werman [20] fuse constraints and measurements using an extended Kalman filter (EKF) by treating constraints as measurements with zero uncertainty. The "preimposed" kinematic chain approach is applied to tracking a robotic arm by Nickels and Hutchinson [21], who use an EKF to recover a state vector of arm joint angles and velocities from point measurements but who simplify matters by assuming a fixed and known base pose. In hand tracking, Rehg and Kanade [22] put joint angles and pose into a nonlinear minimization. In full body tracking, Bregler and Malik [2] develop a linear relationship between instantaneous motion and pose change using exponential representations within a kinematic chain, and Sidenbladh et al. [3] use the kinematics in a generative model of image appearance.

In each of these, the surrounding observation and computation methodologies are sufficiently different to make immediate comparison difficult, and no detailed comparison is available in the literature. Perhaps the two works in the different classes that share most are those of Bregler and Malik [2] and Drummond and Cipolla [10], but even here the projection and image measurement models differ. This paper attempts a fair comparison of these by imposing a common base of image and scene measurement. That base is actually Harris' rather older "RAPiD" tracker [23], a method which (for completeness) we show is wholly equivalent to the subpart tracking stage of Drummond and Cipolla. The kinematic chain method is redesigned ab initio as an articulated RAPiD tracker. A more detailed study is available in [24].

\section{Scene and Projected Image Motion}

In both Drummond and Cipolla's tracker and the articulated $\mathrm{RAPiD}$ tracker, referred to below as DCT and ART, a rigid subpart of an articulated object is described in a object frame 0 by the coordinates $\mathbf{X}$ of each of a set of control points, which may be genuine points on the object, but which more usually are parametrized locations on fixed crease and albedo edges, or are generated on the fly on extremal edges (Fig. 1). To allow multiple, possibly moving, cameras to be treated equally, the object's pose will be one or other representation of the rotation and translation $\left\{\mathrm{R}_{0}^{W}, \mathbf{t}_{0 W}\right\}$ that transform points in frame 0 into a fixed world frame $W$. The position of each camera $\mathrm{C}_{k}$ is defined similarly by $\left\{\mathrm{R}_{W}^{C}, \mathbf{t}_{W C}\right\}_{k}$. It is assumed that external and internal camera calibrations are known.

ART follows Harris' formulation, in which the kinematic screw $\mathbf{s}$ is recovered in the "aligned" frame $A$, whose origin coincides with that of the object but which is aligned with the world frame, such that $\mathbf{X}^{A}=\mathrm{R}_{0}^{W} \mathbf{X}^{0}$. The object's instantaneous velocity in $W$ is

$$
\dot{\mathbf{X}}^{W}=\left(\left[-\mathbf{X}^{A}\right]_{\times} \mid \mathrm{I}_{3}\right)\left(\begin{array}{c}
\omega \\
v
\end{array}\right)=\mathrm{H} \mathbf{s},
$$

where the antisymmetric matrix $[\cdot]_{\times}$generates the vector product and $\mathrm{I}_{3}$ is the $3 \times 3$ identity matrix. The velocity is transformed into a camera frame as $\dot{\mathbf{X}}^{C}=\mathrm{R}_{W}^{C} \mathrm{Hs}$, and projection $\mathbf{x}=\mathbf{X}^{C} / Z^{C}$ into a normalized image (with unit focal length, unit aspect ratio, and origin at the optic centre) gives the image motion as

$$
\dot{\mathbf{x}}=\left(1 / Z^{C}\right)\left[\mathrm{I}_{3}-\mathbf{x}[001]\right] \mathrm{R}_{W}^{C} \text { Hs. }
$$

The equivalence of this approach with that in DCT is easily shown. DCT recovers the 6-vector $\alpha$ of coefficients of the generators of SE(3) describing the change of homogeneous transformation between object and camera. To draw proper comparison, we specify this change in the aligned frame and so it is the 

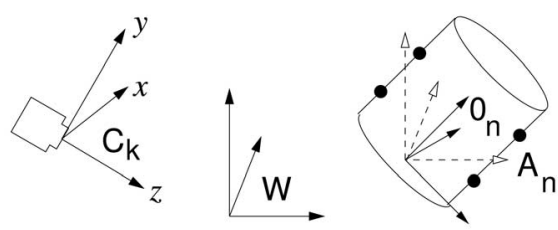

Fig. 1. An object $n$ is modeled within its own coordinate frame $0_{n}$ as a set of control points lying on edges, which may be crease, albedo, or extremal. $\mathrm{C}_{k}, \mathrm{~W}$, and $\mathrm{A}_{n}$ are the $k$ th camera, the world, and $n$th object aligned frames, respectively.

$4 \times 4$ transformation from object to world frames that is updated from $\mathrm{T}_{A}^{W} \mathrm{~T}_{0}^{A}$ to $\mathrm{T}_{A}^{W} \mathrm{MT}_{0}^{A}$ after movement, where $\mathrm{M}=\exp \left(\sum_{i} \alpha_{i} \mathrm{G}_{i}\right)$. To conform with the conventional screw order, we switch the first and last three generators of the Lie group from [10]; in turn, they are associated with angular velocity about and translational velocity in the $x, y$, and $z$ directions. The nonzero elements of the matrices are

$$
\begin{aligned}
& \mathrm{G}_{1}: g_{32}=-g_{23}=1 ; \quad \mathrm{G}_{4}: g_{14}=1 \\
& \mathrm{G}_{2}: g_{13}=-g_{31}=1 ; \quad \mathrm{G}_{5}: g_{24}=1 \\
& \mathrm{G}_{3}: g_{21}=-g_{12}=1 ; \quad \mathrm{G}_{6}: g_{34}=1 .
\end{aligned}
$$

The pose change is small, so $\mathrm{M} \approx \mathrm{I}+\sum_{i} \alpha_{i} \mathrm{G}_{i}$, and the equivalence with (1) becomes apparent:

$$
\begin{aligned}
\dot{\mathbf{X}}^{W} & =\left(\begin{array}{cc}
\mathrm{I} & \mathbf{t}_{0 W} \\
\mathbf{0}^{\top} & 1
\end{array}\right) \sum_{i} \alpha_{i} \mathrm{G}_{i}\left(\begin{array}{cc}
\mathrm{R}_{0}^{W} & \mathbf{0} \\
\mathbf{0}^{\top} & 1
\end{array}\right)\left(\begin{array}{c}
\mathbf{X}_{0} \\
1
\end{array}\right) \\
& =\left(\begin{array}{cc}
\mathrm{I} & \mathbf{t}_{0 W} \\
\mathbf{0}^{\top} & 1
\end{array}\right)\left(\begin{array}{cccc}
0 & -\alpha_{3} & \alpha_{2} & \alpha_{4} \\
\alpha_{3} & 0 & -\alpha_{1} & \alpha_{5} \\
-\alpha_{2} & \alpha_{1} & 0 & \alpha_{6} \\
0 & 0 & 0 & 0
\end{array}\right)\left(\begin{array}{c}
\mathbf{X}_{A} \\
1
\end{array}\right) \\
& =\left(\left[-\mathbf{X}^{A}\right]_{\times} \mid \mathrm{I}_{3 \times 3}\right) \boldsymbol{\alpha} .
\end{aligned}
$$

Full motion vectors $\dot{\mathbf{x}}$ could be used to recover the screw $\mathrm{s}$ (or $\alpha$ ) were point to point matches available, but, in both methods, it is usual to match control points to image edges and the resulting aperture problem requires the measurement of $\dot{\mathbf{x}}$ projected onto a direction $\hat{\mathbf{d}}$ normal to the control point's edge. The measurement equation for control point $i$ is

$$
\left(1 / Z_{i}^{C}\right) \hat{\mathbf{d}}_{i}^{\top}\left[\mathrm{I}_{3}-\mathbf{x}_{i}[001]\right] \mathrm{R}_{W}^{C} \mathrm{H}_{i} \mathbf{s}=\hat{\mathbf{d}}_{i}^{\top} \dot{\mathbf{x}}_{i}
$$

or $\mathbf{f}_{i} \mathbf{s}=d_{i}$, and both methods require stacking six or more measurement rows into $\mathbf{F s}=\mathbf{d}$ for solving with some variant of least squares.

\section{An ARTICULATED RAPID TRACKER: ART}

We propose an extension to Harris' method to articulated objects using a standard kinematical approach. Consider two subparts labeled 0 and 1 , connected by a pure revolute joint with joint angle $\theta_{1}$ located at $\ell_{0}$ in subpart 0 's frame. A point $\mathbf{P}$ at $\mathbf{X}^{1}$ referred to the local frame attached to subpart 1 of the articulated mechanism is at

$$
\mathbf{X}^{0}=\mathrm{T}_{1}^{0} \mathbf{X}^{1}=\left(\begin{array}{cc}
\mathrm{R}\left(\theta_{1}\right) & \boldsymbol{\ell}_{0} \\
\mathbf{0}^{\top} & 1
\end{array}\right) \mathbf{X}^{1}
$$

in part 0 's frame. As point $\mathrm{P}$ is stationary in frame 1, differentiation with respect to time gives

$$
\dot{\mathbf{X}}^{0}=\dot{\theta}_{1}\left(\begin{array}{cc}
\mathrm{R}^{\prime}\left(\theta_{1}\right) & 0 \\
0^{\top} & 0
\end{array}\right) \mathbf{X}^{1}=\dot{\theta}_{1} \mathrm{U}_{1}^{0} \mathbf{X}^{1},
$$

where $R^{\prime}$ is the element-by-element derivative of $R$ with respect to $\theta_{1}$. Similar expressions are available for purely prismatic joints. For a point on subpart $J$ of a mechanism,

$$
\begin{aligned}
& \mathbf{X}^{0}=\mathrm{T}_{1}^{0}\left(\theta_{1}\right) \mathrm{T}_{2}^{1}\left(\theta_{2}\right) \ldots \mathrm{T}_{J}^{J-1}\left(\theta_{J}\right) \mathbf{X}^{J}, \\
& \dot{\mathbf{X}}^{0}=\left(\dot{\theta}_{1} \mathrm{D}_{J 1}^{0}+\dot{\theta}_{2} \mathrm{D}_{J 2}^{0}+\ldots+\dot{\theta}_{J} \mathrm{D}_{J J}^{0}\right) \mathbf{X}^{J},
\end{aligned}
$$

where $\mathrm{D}_{J 1}^{0}=\mathrm{U}_{1}^{0} \mathrm{~T}_{2}^{1} \ldots \mathrm{T}_{J}^{J-1}, \mathrm{D}_{J 2}^{0}=\mathrm{T}_{1}^{0} \mathrm{U}_{2}^{1} \ldots \mathrm{T}_{J}^{J-1}$, and so on. For a mechanism with $(N+1)$ parts, this expression can be written as a linear sum over all $N$ joint velocities $\dot{\theta}=\left(\dot{\theta}_{1} \ldots \dot{\theta}_{N}\right)^{\top}$

$$
\dot{\mathbf{X}}^{0}=\left(\mathbf{a}_{1}\left|\mathbf{a}_{2}\right| \ldots\left|\mathbf{a}_{J}\right| 0_{4 \times(N-J)}\right) \dot{\boldsymbol{\theta}}=A_{4 \times N} \dot{\boldsymbol{\theta}},
$$

where $\mathbf{a}_{j}=\mathrm{D}_{J j}^{0} \mathbf{X}^{J}$. As $\dot{\mathbf{X}}^{0}$ is a direction vector, its fourth component is always zero. Below, we use it as a 3-vector and write $\mathrm{A}$ as a $(3 \times N)$ matrix $\mathrm{A}_{3 \times N}=\left(\mathrm{I}_{3} \mid 0\right) \mathrm{A}_{4 \times N}$.

If the pose of base part 0 is given by $\left\{\mathrm{R}_{0}^{W}, \mathbf{t}_{0 W}\right\}$ referred to the world frame, then the instantaneous velocity in the world frame is

$$
\begin{aligned}
\dot{\mathbf{X}}^{W} & =\mathrm{R}_{0}^{W} \dot{\mathbf{X}}^{0}+\boldsymbol{\omega} \times \mathrm{R}_{0}^{W} \mathbf{X}^{0}+\boldsymbol{v} \\
& =\mathrm{R}_{0}^{W} \mathrm{~A}_{3 \times N} \dot{\boldsymbol{\theta}}+\boldsymbol{\omega} \times \mathbf{X}^{\mathrm{A}}+\boldsymbol{v} .
\end{aligned}
$$

The velocity in the world frame is again $\dot{\mathbf{X}}^{W}=$ Hs, but now $\mathrm{H}$ has $N$ extra columns at the right

$$
\mathrm{H}=\left(\left[-\mathbf{X}^{A}\right]_{\times}\left|\mathrm{I}_{3 \times 3}\right| \mathrm{R}_{0}^{W} \mathrm{~A}_{3 \times N}\right)
$$

and $\mathbf{s}$ is augmented with the joint velocities

$$
\mathbf{s}=\left(\boldsymbol{\omega}^{\top} \boldsymbol{v}^{\top} \dot{\boldsymbol{\theta}}^{\top}\right)^{\top} .
$$

The construction of the pose update equation from the measurements then follows exactly as given in (4).

\subsection{Implementation and Examples}

The articulated RAPiD tracker has been implemented as a video rate process for multiple articulated objects viewed by one or more cameras. The articulated objects are restricted to noncyclic mechanisms and, so, the coupling of subparts with joints can be represented as a tree. To gather the information to complete each pose update, the tree is explored depth first. Denoting the root and current subparts as nodes 0 and $j$, respectively, and the parent of the current node as $p$, the cumulative transformation (5) at the current node is found as $\mathrm{T}_{j}^{0}=\mathrm{T}_{p}^{0} \mathrm{~T}_{j}^{p}$ and stored at the node. To populate (6), the dependency on the joint angle (or length) between parent and current nodes is determined as $\mathrm{D}_{j j}^{0}=\mathrm{T}_{p}^{0} \mathrm{U}_{j}^{p}$, and the dependencies on joint angles (or lengths) $\theta_{j^{\prime}}$ earlier than the current node's parent found as $\mathrm{D}_{j j^{\prime}}^{0}=\mathrm{D}_{p j^{\prime}}^{0} \mathrm{~T}_{j}^{p}$. The D matrices are again stored at the node.

Illustrative output from experiments using three calibrated cameras viewing a $0.5 \times 0.5 \mathrm{~m}^{2}$ working area from near orthogonal directions is shown in Fig. 2. Figs. $2 a$ and $2 b$ show the tracking of a box and hand, the latter with a single articulation for the fingers. The hand and the box are modeled as a single seven dof kinematic tree (three for the hand, plus one for the fingers and three for the box with respect to the hand), constrained to the table plane. When the hand grasps the box, the degrees of freedom between hand and box are switched off and the whole set is tracked as a rigid object, reducing the dimensionality of the problem. Figs. $2 \mathrm{c}$ and $2 \mathrm{~d}$ shows four entities, a plane, mug, ball, and two articulated cylinders, being tracked as one articulated 14 dof object, comprising six dof for the planar object, two each for ball and mug with respect to the plane and four for jointed cylinders.

Mismatching is reduced using robust methods with guided MLESAC [25] selecting control points and least median of squares removing noncollinear measurements from collinear control points. Robust methods are equally applicable to, and necessary for, both methods of applying constraints. We compare the cost implications later. 

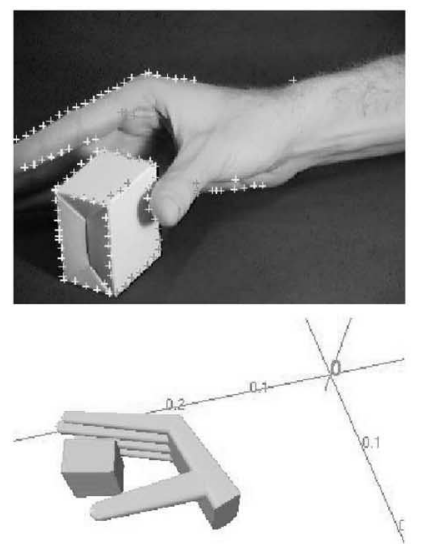

(a)
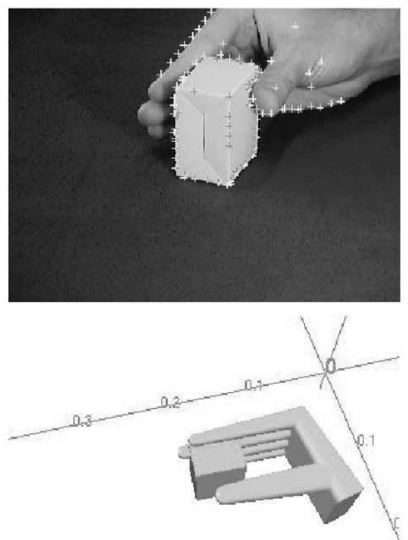

(b)

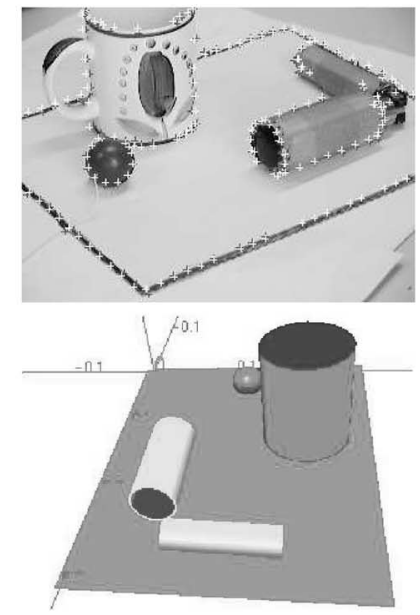

(c)

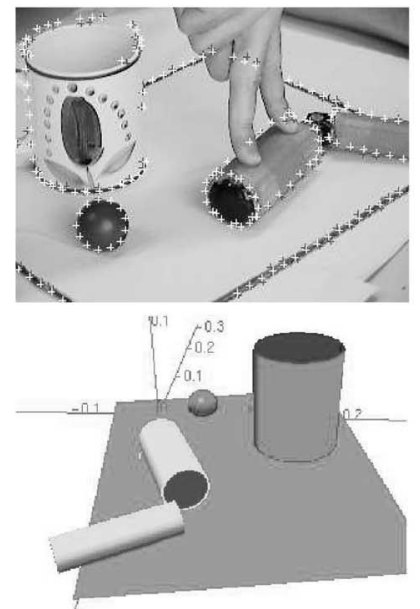

(d)

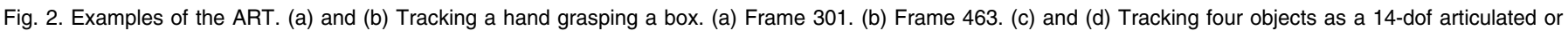
"motion-constrained" object. (c) Frame 3. (d) Frame 4106.

\section{Enforcing Constraints after Measurement}

In Drummond and Cipolla's method [10], the measurement system $\mathbf{F} \alpha=\mathbf{d}$ for each individual subpart is similar to that developed earlier, but the pose adjustment takes place in the object frame, not the aligned frame. The image measurements $d$ are identical with those derived earlier, but the rows $\mathbf{f}$ of $\mathrm{F}$ are generated using

$$
\dot{\mathbf{X}}^{W}=\left(\begin{array}{cc}
\mathrm{R}_{0}^{W} & \mathbf{t}_{0 W} \\
0^{\top} & 1
\end{array}\right) \sum_{i} \alpha_{i} \mathrm{G}_{i}\left(\begin{array}{c}
\mathbf{X}_{0} \\
1
\end{array}\right) .
$$

When the constraints are applied, each subpart's unconstrained value of $\alpha$ is modified to $\beta$. The sum-squared fitting cost rises to $S_{\beta}=(\mathrm{F} \beta-\mathbf{d})^{\top}(\mathrm{F} \beta-\mathbf{d})$ and, hence, the extra cost is

$$
S_{\beta}-S_{\alpha}=(\beta-\alpha)^{\top}\left[\mathrm{F}^{\top} \mathrm{F}\right](\boldsymbol{\beta}-\boldsymbol{\alpha}) .
$$

A constraint between two parts $p$ and $q$ implies equivalence between corresponding screw values, when referred to the same frame. Drummond and Cipolla write each constraint as

$$
\left(\boldsymbol{\beta}_{p}-\beta_{q}^{p}\right)^{\top} \mathbf{c}_{p, q}^{k}=0,
$$

where the superscript $p$ denotes a value referred to the $p$ th coordinate frame. For each of $k=1, \ldots, K_{p, q}$ constraints between $p$ and $q$, the $\mathbf{c}_{p, q}^{k}$ is a column 6-vector drawn from the identity matrix $\mathrm{I}_{6}$. Selecting columns 1-3 constrains $\omega_{x, y, z}$, respectively, and selecting columns $4-6$ constrains $v_{x, y, z}$. If $K_{p, q}$ is 0 or 6 , the joint is broken or rigid.

\subsection{Handling General Trees of Subparts in DCT}

In [10], Drummond and Cipolla describe the solution for two subparts connected by a hinge and, in [7], they sketch a solution for a chain of subparts. However, for comparison with the articulated RAPiD tracker, it is necessary properly to understand how DCT might handle branching in the kinematic chain.

As coordinate frames $p$ and $q$ are related by $\mathbf{X}^{p}=\mathrm{T}_{q}^{p} \mathbf{X}^{q}$, the screw $\beta_{q}^{p}$ is given by $\beta_{q}^{p}=\operatorname{Ad}\left(\mathrm{T}_{q}^{p}\right) \beta_{q}$, where the adjoint transformation is abbreviated below to $\mathcal{T}_{q}^{p} \equiv \operatorname{Ad}\left(\mathrm{T}_{q}^{p}\right)$. A constraint can therefore be rewritten as $\left[\boldsymbol{\beta}_{p}-\mathcal{T}_{q}^{p} \boldsymbol{\beta}_{q}\right]^{\top} \mathbf{c}_{p, q}^{k}=0$. Now, consider $N+$ 1 subparts arranged in an articulated tree. The DCT problem becomes one of finding

$$
\min _{\boldsymbol{\beta}_{q}} \sum_{q}\left(\boldsymbol{\beta}_{q}-\boldsymbol{\alpha}_{q}\right)^{\top} \mathrm{F}_{q}^{\top} \mathrm{F}_{q}\left(\boldsymbol{\beta}_{q}-\boldsymbol{\alpha}_{q}\right)
$$

subject to sets of motion constraint equations. Each subpart with children generates one constraint set for each of its children, so that the subpart $q$ shown in Fig. 3a with ancestors $p, p^{-}$, etc., and with possible multiple lines of descendants $a, a^{+}$, etc., $b, b^{+}$, etc., is referenced by the following constraint sets:

$$
\begin{array}{lll}
(p, q) & \text { If parent } p \text { exists : } & {\left[\boldsymbol{\beta}_{p}-\mathcal{T}_{q}^{p} \boldsymbol{\beta}_{q}\right]^{\top} \mathbf{c}_{p, q}^{k}=0} \\
(q, a) & \text { If } 1 \text { st child } a \text { exists : } & {\left[\boldsymbol{\beta}_{q}-\mathcal{T}_{a}^{q} \boldsymbol{\beta}_{a}\right]^{\top} \mathbf{c}_{q, a}^{k}=0} \\
(q, b) & \text { If } 2 \text { nd child } b \text { exists : } & {\left[\boldsymbol{\beta}_{q}-\mathcal{T}_{b}^{q} \boldsymbol{\beta}_{b}\right]^{\top} \mathbf{c}_{q, b}^{k}=0}
\end{array}
$$

and so on if $q$ has further children.

That part of the Lagrange system depending of differentiation with respect to $\beta_{q}$ is therefore

$$
2 \mathrm{C}_{q}\left(\boldsymbol{\beta}_{q}-\boldsymbol{\alpha}_{q}\right)-\sum_{m=1}^{K_{p, q}} \lambda_{p, q}^{m} \mathcal{T}_{q}^{p^{\top}} \mathbf{c}_{p, q}^{m}+\sum_{m=1}^{K_{q, a}} \lambda_{q, a}^{m} \mathbf{c}_{q, a}^{m}+(\cdot)=\mathbf{0}_{6},
$$

where the first term derives from the cost, where the $\lambda$ values are the Lagrange multipliers, and where $\mathrm{C}_{q}=\mathrm{F}_{q}^{\top} \mathrm{F}_{q}$. The first summation is omitted if $q$ has no parent, the second if $q$ has no children, and extra terms of the form $\sum_{m=1}^{K_{q, b}} \lambda_{q, b}^{m} \mathbf{c}_{q, b}^{m}$ are added for further children $b$, and so on. Rearranging,

$$
\boldsymbol{\beta}_{q}=\boldsymbol{\alpha}_{q}+\sum_{m=1}^{K_{p, q}} \lambda_{p, q}^{m} \frac{1}{2} \mathrm{C}_{q}^{-1} \mathcal{T}_{q}^{p \top} \mathbf{c}_{p, q}^{m}-\sum_{m=1}^{K_{q, a}} \lambda_{q, a}^{m} \frac{1}{2} \mathrm{C}_{q}^{-1} \mathbf{c}_{q, a}^{m}-(\cdot) .
$$

Similar expressions can be written for the other $\beta$ vectors. Replacing all the $\beta$ vectors in the $(p, q)$ constraint set gives

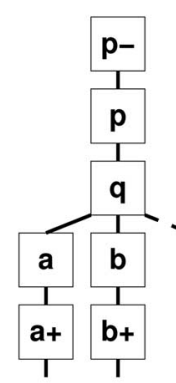

(a)

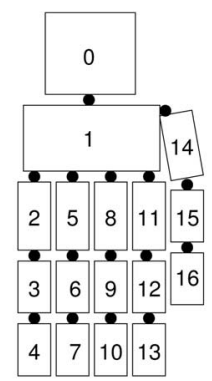

(b)

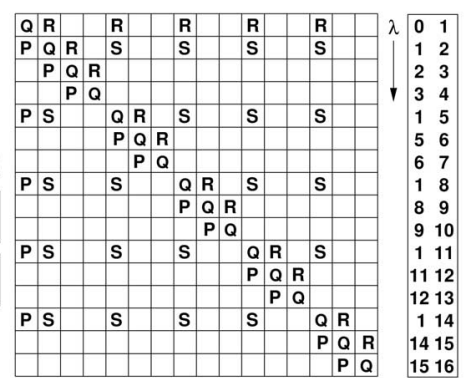

(c)
Fig. 3. (a) General branching model. (b) The hand model and (c) the block structures of its corresponding matrix and $\lambda$ subscripts. 


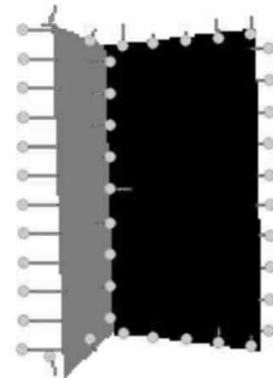

(a)

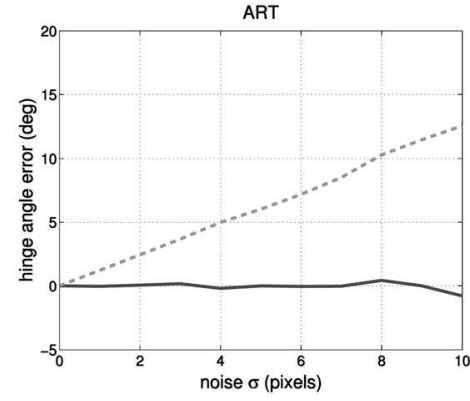

(b)

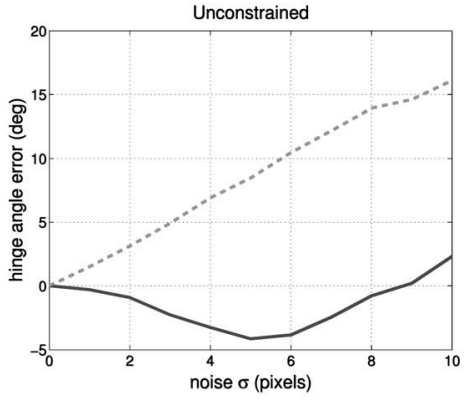

(c)

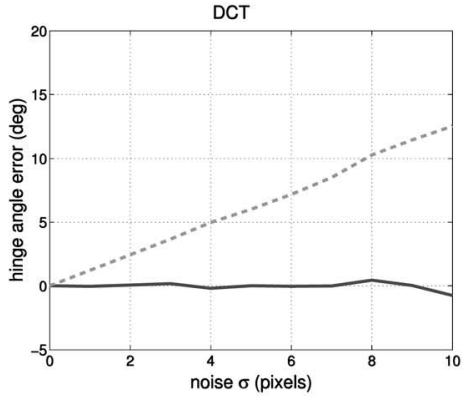

(d)

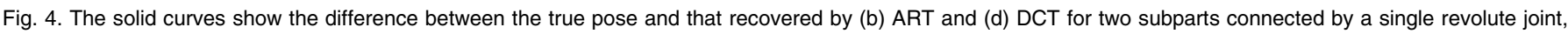

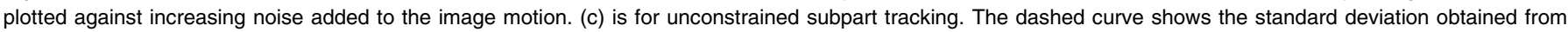
repeated trials.

$$
\begin{aligned}
\sum_{m=1}^{K_{p^{-}, p}} \lambda_{p^{-}, p}^{m} \mathbf{c}_{p, q}^{k}{ }^{\top} \mathrm{C}_{p}^{-1} \mathcal{T}_{p}^{p^{-\top}} \mathbf{c}_{p^{-}, p}^{m} & \\
& -\sum_{m=1}^{K_{p, q}} \lambda_{p, q}^{m} \mathbf{c}_{p, q}^{k}{ }^{\top}\left[\mathrm{C}_{p}^{-1}+\mathcal{T}_{q}^{p} \mathrm{C}_{q}^{-1} \mathcal{T}_{q}^{p \top}\right] \mathbf{c}_{p, q}^{m} \\
& +\sum_{m=1}^{K_{q, a}} \lambda_{q, a}^{m} \mathbf{c}_{p, q}^{k}{ }^{\top} \mathcal{T}_{q}^{p} \mathrm{C}_{q}^{-1} \mathbf{c}_{q, a}^{m}+(. .) \\
& =2 \mathbf{c}_{p, q}^{k}{ }^{\top}\left[\mathcal{T}_{q}^{p} \boldsymbol{\alpha}_{q}-\boldsymbol{\alpha}_{p}\right] \quad k=1, \ldots, K_{p, q},
\end{aligned}
$$

where $(. \cdot)=\sum_{m=1}^{K_{q, b}} \lambda_{q, b}^{m} \mathbf{c}_{p, q}^{k}{ }^{\top} \mathcal{T}_{q}^{p} \mathbf{C}_{q}^{-1} \mathbf{c}_{q, b}^{m}+\ldots$ allows for further children. Similarly, replacing all those in the $(q, a)$ constraint set gives

$$
\begin{aligned}
\sum_{m=1}^{K_{p, q}} \lambda_{p, q}^{m} \mathbf{c}_{q, a}^{k}{ }^{\top} \mathrm{C}_{q}^{-1} \mathcal{T}_{q}^{p^{\top}} \mathbf{c}_{p, q}^{m} \\
\quad-\sum_{m=1}^{K_{q, a}} \lambda_{q, a}^{m} \mathbf{c}_{q, a}^{k}{ }^{\top}\left[\mathrm{C}_{q}^{-1}+\mathcal{T}_{a}^{q} \mathrm{C}_{a}^{-1} \mathcal{T}_{a}^{q \top}\right] \mathbf{c}_{q, a}^{m} \\
+\sum_{m=1}^{K_{a, a+}^{+}} \lambda_{a, a^{+}}^{m} \mathbf{c}_{q, a}^{k}{ }^{\top} \mathcal{T}_{a}^{q} \mathrm{C}_{a}^{-1} \mathbf{c}_{a, a^{+}}^{m}+(\cdot \cdot) \\
=2 \mathbf{c}_{q, a}^{k}{ }^{\top}\left[\mathcal{T}_{a}^{q} \boldsymbol{\alpha}_{a}-\boldsymbol{\alpha}_{q}\right] \quad k=1, \ldots, K_{q, a},
\end{aligned}
$$

with $(. \cdot)=\sum_{m=1}^{K_{q, b}} \lambda_{q, b}^{m} \mathbf{c}_{q, a}^{k}{ }^{\top} \mathrm{C}_{q}^{-1} \mathbf{c}_{q, b}^{m}+\ldots$ Note that if there are multiple children, $b$ and so on, expressions similar to (10) must be added for the $(q, b)$ constraint set, involving swapping $a \leftrightarrow b$.

These expressions are more compactly expressed as

$$
\begin{gathered}
\mathrm{P}_{p, q} \lambda_{p^{-}, p}+\mathrm{Q}_{p, q} \lambda_{p, q}+\mathrm{R}_{p, q}^{a} \lambda_{q, a}\left(+\mathrm{R}_{p, q}^{b} \lambda_{q, b}+\ldots\right)=\mathbf{l}_{p, q}, \\
\mathrm{P}_{q, a} \lambda_{p, q}+\mathrm{Q}_{q, a} \lambda_{q, a}+\mathrm{R}_{q, a}^{a+} \lambda_{a, a^{+}}\left(+\mathrm{S}_{q, a}^{b} \lambda_{q, b}+\ldots\right)=\mathbf{l}_{q, a},
\end{gathered}
$$

which contribute to a system $\mathrm{M} \lambda=1$ for solution. Once the $\lambda$ are known, the constrained screws $\beta_{q}$ are derived for each subpart from (8). The bracketed terms are used for additional children, and a further equation of the form of (12) is generated for each additional child, with $a \leftrightarrow b, a \leftrightarrow c$, etc. The $k$ th row and $m$ th column of the various quantities are

$$
\begin{aligned}
\mathbf{l}_{p, q}(k) & =2 \mathbf{c}_{p, q}^{k}{ }^{\top}\left[\mathcal{T}_{q}^{p} \boldsymbol{\alpha}_{q}-\boldsymbol{\alpha}_{p}\right], \\
\mathrm{P}_{p, q}(k, m) & =\mathbf{c}_{p, q}^{k}{ }^{\top} \mathrm{C}_{p}^{-1} \mathcal{T}_{p}^{p^{-\top}} \mathbf{c}_{p^{-}, p}^{m}, \\
\mathbf{Q}_{p, q}(k, m) & =-\mathbf{c}_{p, q}^{k}{ }^{\top}\left[\mathrm{C}_{p}^{-1}+\mathcal{T}_{q}^{p} \mathrm{C}_{q}^{-1} \mathcal{T}_{q}^{p \top}\right] \mathbf{c}_{p, q}^{m}, \\
\mathrm{R}_{p, q}^{a}(k, m) & =\mathbf{c}_{p, q}^{k}{ }^{\top} \mathcal{T}_{q}^{p} \mathrm{C}_{q}^{-1} \mathbf{c}_{q, a}^{m}, \\
\mathrm{~S}_{q, a}^{b}(k, m) & =\mathbf{c}_{q, a}^{k} \mathrm{C}_{q}^{-1} \mathbf{c}_{q, b}^{m} .
\end{aligned}
$$

We remark on three configurations. First, when the subparts are arranged as a linear chain, labeled here from 0 to $N$, the system is block tridiagonal in PQR for which an $O(n)$ method exists to recover the $\lambda$ values without explicitly building or inverting the matrix $M$. Second, when branching occurs, the system loses its block tridiagonal form and its structure depends on the object's structure. By way of example, the structure in Fig. 3b generates the system in Fig. $3 \mathrm{c}$ for solution. The structure is block symmetric and sparse, but can no longer be solved in $O(n)$ time. Third, cyclic constraints generate non-block-symmetric $M$ matrices whose rank needs monitoring to avoid noise imposing spurious rigidity.

\section{EXPERIMENTAL COMPARISON}

\subsection{Similarity of Results}

Despite their very different constraint formulations, both trackers are single-shot linear methods, and, given that relationships between scene and image have been shown to be identical, one should expect them to give the same results. To verify this against known ground truth, a CAD model of two hinged subparts was generated and both trackers deployed on the resulting imagery as the hinged opened between successive frames. Repeated trials were performed with increasing amounts of zero-mean Gaussian noise added to the image motion. Fig. 4a shows a typical view and match set. The lower traces in Figs. $4 \mathrm{~b}, 4 \mathrm{c}$, and $4 \mathrm{~d}$ show the difference between the veridical hinge angle and that recovered using ART, subpart tracking without constraint, and DCT after turning on the constraint. It is evident that, as soon as the constraints are applied in DCT, the modified angle becomes all but identical with that recovered using built-in kinematic constraints. Indeed, the results from the two methods differed by at most parts in $10^{5}$, effectively at the limits of expected numerical accuracy. The recovered standard deviations, the upper traces in Figs. $4 \mathrm{~b}$ and $4 \mathrm{~d}$, are also identical.

\subsection{Computational Cost}

Fig. 5 shows comparisons of the times for a single update cycle of the core operations of ART and DCT run on a 1.8 GHz Pentium 4. Times were accumulated over many trials, with each data point taking at least $20 \mathrm{~s}$ to collect, giving each datum the same fractional error of order $10^{-2}$ percent. The object is taken to have $n$ subparts with $p$ control points per subpart and is made up of a single articulated chain. Fig. 5a shows that, with up to 10 subparts, there is negligible difference between the methods and that, at 30 subparts, the time taken by ART is about twice that by DCT. Up to 100 parts, the cost in ART is still dominated by $O\left(n^{2}\right)$, but beyond (not shown) it does become $O\left(n^{3}\right)$ as expected for the Cholesky decomposition of the $(n+5)^{2}$ matrix $\mathrm{F}^{\top} \mathrm{F}$ that occurs in the least squares solution of $\mathrm{Fs}=$ d. Fig. $5 \mathrm{~b}$ shows that both methods scale predominantly linearly with the number of control points $p$ per link. 


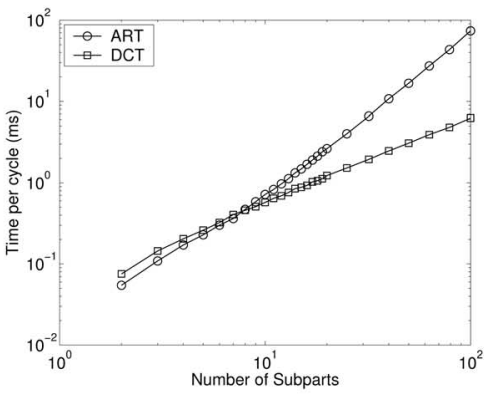

(a)

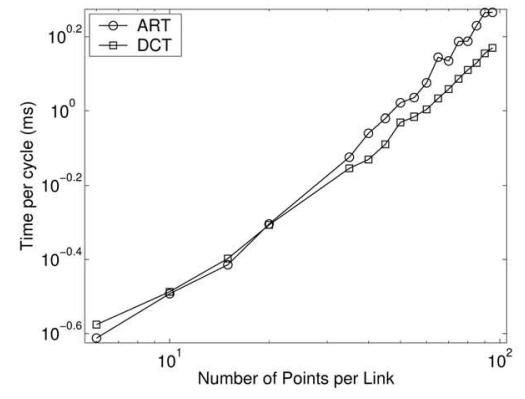

(b)

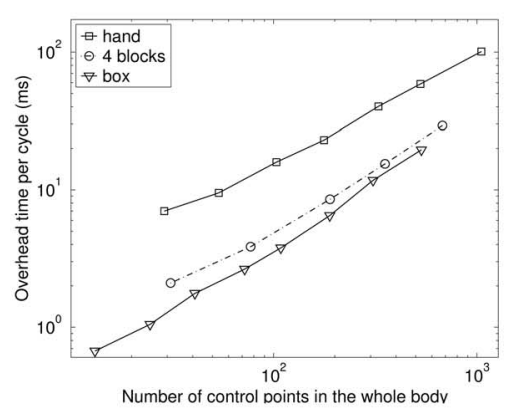

(c)

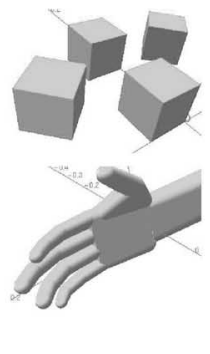

(d)

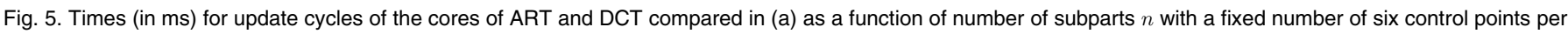

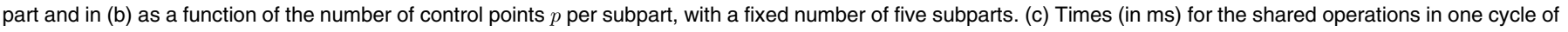
ART and DCT, as a function of the average number of visible control points in the whole body. (d) The "4 blocks" and "hand" models used in (c).

Fig. $5 \mathrm{c}$ shows the computation time of operations that are shared by both trackers, including occlusion handling, projection of control points, image operations, and related operating system overhead. Three objects of increasing complexity were used: a single part box with six dof, an object with four parts and nine dof, and a hand with forearm model with 17 parts and 28 dof. The tests used $320 \times 240$ images acquired from three cameras. The search range from control points was \pm 10 pixels. The number of potential control points was increased in steps but, because of occlusion, not all the control points are always visible and the average visible number for a sequence is used as the abscissa. The time increase is dominated by a linear dependence on the number of visible control points.

For all but small problems, DCT is faster than ART and its linear behavior with number of parts demands its use on large problems, say, those involving more than 100 subparts. However, for modest numbers of subparts, the timing differences are insignificant compared with the equally shared costs. As examples, the different cores of DCT and ART took $0.45 \mathrm{~ms}$ and $0.41 \mathrm{~ms}$, respectively, to run on the four-block object with 100 control points, but shared operations took some $6 \mathrm{~ms}$; on the hand model with some 170 control points ART's time was $2.5 \mathrm{~ms}$, but the shared operations took over $13 \mathrm{~ms}$.

The core times given for DCT are so far those for simple chains where the tridiagonal system is solved. On a chain with the same number of parts as the hand and, again, 170 control points, DCT took some $1.2 \mathrm{~ms}$, but solving the actual branched system shown in Figs. $3 \mathrm{~b}$ and $3 \mathrm{c}$ took some 2.5 times longer, a little slower than ART. Once any branching occurs, we find that that general matrix solvers give a solution time dependent mostly on the number of subparts, not the degree of branching; for example, with 16 parts, two chains of eight takes the same time as four chains of four.

\subsection{Algorithmic Robustness}

In general, tracking unconstrained subparts is certainly more fragile than tracking subparts collectively in a constrained system. The more degrees of freedom, the greater the likelihood of fitting to noise. Breakage might occur when, for example, the available control points do not provide sufficient constraint-a cylinder with points only along its extremal boundaries is an example-or when a subpart moves quickly at the end of a long linkage. On first consideration, this seems to make DCT inherently less robust than ART, but this is not the case. Assume that, at the start of an update cycle, both trackers have placed their subparts in the same location. They will generate the same control points and, therefore, generate the same measurements.

Suppose first that there are enough measurements to determine the pose of each subpart, but that the measurements belonging to a particular subpart are quite erroneous. Using ART, the costs are immediately shared and the pose updates of all the subparts will be disturbed somewhat, whereas, in DCT, the initial pose updates $(\alpha)$ will all be better, except for the erroneous one. However, when the constraints are imposed, the costs of (mis)fitting with the $\beta$ values are all correctly accounted for and minimized. Because the measurements are the same, the solutions must be same.

Suppose now that there are insufficient measurements to determine the pose update of a particular subpart. It is important not to discard those measurements that are available, but instead invent a pose update $\alpha$ for that subpart against which the change in cost when using some $\beta$ instead may be measured, a change which could easily now be a decrease rather than the usual increase. This tactic extends to zero measurements, where the cost change when moving the part is zero. However, what is lost in this case is the ability to separate the recovery of the Lagrange multipliers and the $\beta$ values. Insufficient measurements means that $\mathrm{C}=\mathrm{F}^{\top} \mathrm{F}$ is rank deficient and, so, cannot be inverted in (8). There appears no generalizable alternative to solving the linear system in its entirety, with a stacked vector of $\beta$ and $\lambda$ vectors as the unknowns.

\subsection{Data Robustness}

To consider the likely cost of computing the solutions to ART and DCT in a robust manner using a random sampler, we use the relationship $P=1-\left(1-\psi^{m}\right)^{I}$ for the confidence $P$ that a valid minimal set of $m$ measurements will be selected after $I$ trials when the fraction of valid data is $\psi$. Though spatial and other considerations usually make this expression an underestimate for the number of trials, the comparative results will be less affected. For $n$ subparts with $(n-1) 1$ dof joints, the trials required are

$$
I_{\mathrm{ART}}=\frac{\log (1-P)}{\log \left(1-\psi^{5+n}\right)} ; \quad I_{\mathrm{DCT}}=n \frac{\log (1-P)}{\log \left(1-\psi^{6}\right)} .
$$

Multiplying these expressions by the different time costs per iteration of each method gives the pairs of curves in Fig. 6 for 10, 20 , and 30 subparts derived at $P=95$ percent confidence and each plotted as a function of the percentage of outlying or invalid data, $100(1-\psi)$. Also shown is the locus of the crossover point, as the number of parts is varied. For a modest number of parts and quite low values of corruption, ART's requirements undercut DCT's, but more remarkable is how rapidly a certain fraction of outliers becomes intolerable as the number of subparts rises.

\section{Concluding Remarks}

The performance of two methods of imposing constraints on articulated motion during tracking has been compared. Notwithstanding the very different ways in which the constraints are 


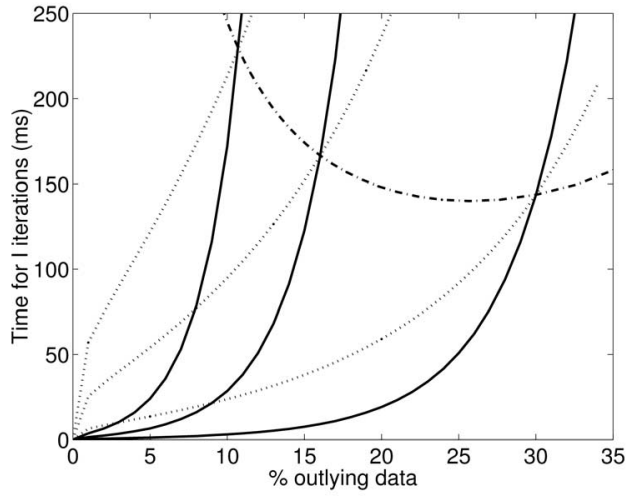

Fig. 6. Pairs of curves showing the times required to complete the required random sampling trials as the percentage of invalid, outlying data varies. The pairs of curves are for 30 (left), 20 (center), and 10 (right) subparts and, in each pair, the solid and dotted lines are from ART and DCT, respectively. Also shown is the locus of curve pair intersections.

applied, results for the pose updates have been demonstrated to be identical. As both are performing linear single-shot pose updates, this is expected.

The significant advantage of Drummond and Cipolla's approach, and this applies generally to postimposed methods, is that, when applied to simple chains, they retain $O(n)$ complexity in the number of subparts $n$. In constrast, we find that ART rises from $O(n)$ for low $n$, to $O\left(n^{2}\right)$ for $30<n<100$ before eventually succumbing to $O\left(n^{3}\right)$. However, for tens of subparts, the absolute difference in core cost is substantially outweighed by the commonly shared costs of model projection, image search, and so on.

A less satisfactory aspect of postimposed methods is that the less complicated the object's kinematics, the more computation has to be done to impose the constraints, an outcome of allowing the degrees of freedom to grow to their maximum. While in DCT this extra effort is very neatly tamed if the object comprises a chain of subparts, other configurations become harder to manage. During branching, for example, when a subpart must satisfy more than one set of constraints, the system no longer has a tridiagonal structure. The block-symmetric structure it acquires instead depends on the model's structure, but no general fast solution methods exist. Indeed, in [7], Drummond and Cipolla adopt an iterative solution. This element of algorithmic frailty appears again when there is insufficient information to solve for the initial pose update of a particular part. However, this disadvantage is mitigated by the postimposed methods' ability to handle more complex constraint regimes.

Although the preimposed method has an advantage over the postimposed method in its time required to complete trials of a random sampler, the more interesting observation is the sharp increase in time for both with rising number of parts. It is better to improve the quality of measurement than to rely on random sampling to clean up afterwards.

What more general conclusion can be drawn about representing multiple articulated objects? The broad similarity in cost for modest number of subparts and the more predictable nature of the computation of the constraints suggest that individual articulated objects might best use preimposed constraints and that postimposed constraints be used to account for the motion of objects which make contact and later break apart. As contact approaches between two parts, tracking performance with and without the relevant constraints can be tested to decide whether the motion was constrained or still independent. The most significant aspect of postimposed methods is that they make the switching on and off of constraints easy, after, and quite separately from, the expensive process of making image measurements.

\section{ACKNOWLEDGMENTS}

This work was supported by Grant GR/N03266 from the UK Engineering and Physical Science Research Council and by Brazilian Government CAPES scholarship to TEdC.

\section{REFERENCES}

[1] D.C. Hogg, "Model-Based Vision: A Program to See a Walking Person," Image and Vision Computing, vol. 1, no. 1, pp. 5-20, 1983.

[2] C. Bregler and J. Malik, "Tracking People with Twists and Exponential Maps," Proc. IEEE Conf. Computer Vision and Pattern Recognition, pp. 8-15, 1998.

[3] H. Sidenbladh, M.J. Black, and D.J. Fleet, "Stochastic Tracking of 3D Humand Figures Using 2D Image Motion," Proc. Sixth European Conf. Computer Vision, pp. 702-718, 2000.

[4] S.X. Ju, M.J. Black, and Y. Yacoob, "Cardboard People: A Parametrized Model of Articulated Motion," Proc. Second Int'l Conf. Automatic Face and Gesture Recognition, pp. 38-44, 1996.

[5] D.M. Gavrila and L.S. Davis, "3D Model-Based Tracking of Humans in Action: A Multiview Approach," Proc. IEEE Conf. Computer Vision and Pattern Recognition, pp. 73-80, 1996.

[6] B. Stenger, P.R.S. Mendonca, and R. Cipolla, "Model-Based 3D Tracking of an Articulated Hand," Proc. IEEE Conf. Computer Vision and Pattern Recognition, vol. 2, pp. 310-315, 2001.

[7] T. Drummond and R. Cipolla, "Real-Time Tracking Highly Articulated Structures in the Presence of Noisy Measurements," Proc Eighth Int'l Conf. Computer Vision, 2001.

[8] T. Heap and D. Hogg, "Towards 3D Hand Tracking Using a Deformable Model," Proc. Second Conf. Face and Gesture Recognition, pp. 140-145, Oct. 1996.

[9] J. Deutscher, A. Blake, and I.D. Reid, “Articulated Body Motion Capture by Annealed Particle Filtering," Proc. Conf. Computer Vision and Pattern Recognition, pp. 2126-2133, 2000.

[10] T. Drummond and R. Cipolla, "Real-Time Visual Tracking of Complex Structures," IEEE Trans. Pattern Analysis and Machine Intelligence, vol. 24, no. 7, pp. 932-946, July 2002.

[11] M. Brand, "Shadow Puppetry," Proc. Seventh IEEE Int'l Conf. Computer Vision, pp. 1237-1244, 1999.

[12] B.J. Tordoff, W.W. Mayol, T.E. de Campos, and D.W. Murray, "Head Pose Estimation for Wearable Robot Control," Proc. British Machine Vision Conf. pp. 807-816, 2002.

[13] Y. Yacoob and L.S. Davis, "Learned Temporal Models of Image Motion," Proc. Sixth IEEE Int'l Conf. Computer Vision, pp. 446-453, 1998

[14] S. Wachter and H-H. Nagel, "Tracking Persons in Monocular Image Sequences," Computer Vision and Image Understanding, vol. 74, no. 3, pp. 174192, 1999.

[15] M. Isard and A. Blake, "Contour Tracking by Stochastic Propagation of Conditional Density," Proc. Fourth European Conf. Computer Vision, pp. 343356,1996

[16] C. Sminchisescu and W. Triggs, "Covariance Scaled Sampling for Monocular 3D Body Tracking," Proc. IEEE Conf. Computer Vision and Pattern Recognition, pp. 447-454, 2001.

[17] J.M. Rehg, D.D. Morris, and T. Kanade, "Ambiguities in Visual Tracking of Articulated Objects Using Two- and Three-Dimensional Models," Int'l J. Robotics Research, vol. 22, no. 6, pp. 393-418, 2003.

[18] T. Drummond and R. Cipolla, "Real-Time Tracking of Multiple Articulated Structures in Multiple Views," Proc. Sixth European Conf. Computer Vision, pp. 20-36, 2000.

[19] Y. Wu, G. Hua, and T. Yu, "Tracking Articulated Body by Dynamic Markov Network," Proc. Ninth Int'l Conf Computer Vision, pp. 1094-1101, 2003.

[20] Y. Hel-Or and M. Werman, "Constraint Fusion for Recognition and Localization of Articulated Objects," Int'l J. Computer Vision, vol. 19, no. 1, pp. 15-28, July 1996

[21] K. Nickels and S. Hutchinson, "Model-Based Tracking of Complex Articulated Objects," IEEE Trans. Robotics and Automation, vol. 17, no. 1, pp. 28-36, 2001.

[22] J.M. Rehg and T. Kanade, "Model-Based Tracking of Self-Occluding Articulated Objects," Proc. Fifth IEEE Int'l Conf. Computer Vision, pp. 612617, 1995.

[23] C. Harris, "Tracking with Rigid Models," Active Vision, A. Blake and A. Yuille, eds., pp. 59-73, Cambridge, Mass.: MIT Press, 1992.

[24] T.E. de Campos, B.J. Tordoff, and D.W. Murray, "Linear Recovery of Articulated Pose Change: Comparing Pre- and Post-Imposed Constraints," Technical Report OUEL 2279/05, Dept. of Eng. Science, Univ. of Oxford, 2005.

[25] B.J. Tordoff and D.W. Murray, "Guided Sampling and Consensus for Motion Estimation," Proc. Seventh European Conf. Computer Vision, pp. 82-96, 2002.

$\triangleright$ For more information on this or any other computing topic, please visit our Digital Library at www.computer.org/publications/dlib. 